\title{
PRODUÇÃO DE SERAPILHEIRA EM DIFERENTES FASES SUCESSIONAIS DE UMA FLORESTA SUBTROPICAL SECUNDÁRIA, EM ANTONINA, PR
}

\author{
Kauana Melissa Cunha Dickow ${ }^{1}$, Renato Marques², Carolina Benghi Pinto ${ }^{3}$, Hubert Höfer ${ }^{4}$
}

(recebido: 12 de maio de 2010; aceito: 28 de outubro de 2011)

RESUMO: O presente estudo foi realizado na Reserva Natural Rio Cachoeira, em Antonina (PR), e teve como objetivo principal avaliar a produção de serapilheira em áreas de floresta secundária em diferentes fases de sucessão (fases inicial, média e avançada). As coletas foram realizadas a cada 3 semanas, durante quatro anos (2004 a 2007), em coletores circulares de 0,25 m², distribuídos em número de dois em cada parcela de $100 \mathrm{~m}^{2}$. No total foram utilizados 60 coletores em uma área total de $3000 \mathrm{~m}^{2}$. A serapilheira coletada foi secada e triada em frações (folhas, ramos, órgãos reprodutivos e miscelânea). Dentro da fração folhas, foram selecionadas algumas espécies para estudo específico da contribuição destas à serapilheira total. A produção anual média da serapilheira total

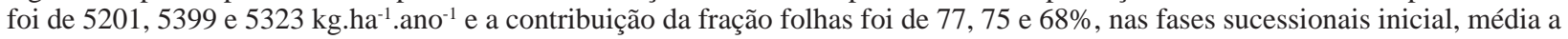
avançada, respectivamente. Na serapilheira foliar da fase inicial predominou a espécie Tibouchina pulchra (jacatirão) (75\%), sendo que nas fases média e avançada não ocorreu o predomínio de apenas uma espécie. A produção de ramos e órgãos reprodutivos não diferiu estatisticamente entre as diferentes fases sucessionais. A produção da fração miscelânea foi maior na fase avançada e não diferiu entre as fases inicial e média. Em geral, foram pequenas as diferenças na produção de serapilheira ao longo do processo sucessional na área do estudo.

Palavras-chave: Ciclagem de nutrientes, regeneração natural, Floresta Atlântica.

\section{LITTER PRODUCTION IN DIFFERENT SUCCESSIONAL STAGES OF A SUBTROPICAL SECONDARY RAIN FOREST, IN ANTONINA, PR}

\begin{abstract}
This study was conducted in the Cachoeira River Natural Reserve, in Antonina, Paraná state, Brazil. The main goal was to assess the litter production of secondary tropical rain forests in different sucessional stages (initial, medium and advanced stages). The litter fall was collected every 3 weeks during four years (2004 to 2007), in circular litter traps of 0.25 ${ }^{2}$, distributed in 30 plots of $100 \mathrm{~m}^{2}$ of area. A total of 60 litter traps were installed in an area of $3000 \mathrm{~m}^{2}$. The litter fall collected at each retrieval date was dried, separated into fractions (leaves, twigs, reproductive organs and miscellaneous) and weighed. Leaves of some tree species were selected from the total litter for a specific study of their contribution to the total litter. The average annual litter production for the three years was 5201, 5399 and $5323 \mathrm{~kg} \cdot \mathrm{ha}^{-1}$.year ${ }^{-1}$, and the percentage contribution of the leaf fraction was 77, 75 and 68\%, in the initial, medium and advanced sucessional stages, respectively. The leaf litter fraction produced in the initial stage was dominated by the species Tibouchina pulchra (jacatirão) (75\%), but in the medium and advanced stages the dominance of only one tree species in leaf litter fraction did not occur. The production of twigs and reproductive organs did not differ statistically among the sucessional stages. The production of miscellaneous fraction was higher in the advanced stage and did not differ between the initial and medium stages. In general, the differences in litter production were little along the sucessional stage in the area of the study.
\end{abstract}

Key words: Nutrient cycling, natural regeneration, Atlantic Rain Forest.

\section{INTRODUÇÃO}

A Serra do Mar é a região mais conservada da Floresta Atlântica, com 36,5\% da sua vegetação original e o segundo maior fragmento está localizado na zona costeira do Estado do Paraná, com quase 509 mil ha de extensão (RIBEIRO et al., 2009). Embora a Floresta Atlântica no Paraná represente um dos melhores remanescentes da formação em termos de conservação no Brasil, diversas áreas de serras e planícies estão recobertas atualmente por formações secundárias em diferentes estádios sucessionais (SILVA, 2003). Além disso, as florestas, em fases iniciais de sucessão nas terras abandonadas, não estão protegidas pelas leis ambientais brasileiras (METZGER, 2009) e, por isso, estão amplamente sujeitas a novas supressões.

\footnotetext{
${ }^{1}$ Engenheira Florestal, Professora Dra. em Engenharia Florestal - Universidade Estadual do Centro-Oeste - PR 153, Km 7, Riozinho - 84500-000 Irati, PR - kauanadickow@gmail.com

${ }^{2}$ Engenheiro Florestal, Professor Dr. em Ciências Florestais - Departamento de Solos e Engenharia Agrícola - Universidade Federal do Paraná Rua dos Funcionários, nº 1540 - 80.035-050 - Curitiba, PR - rmarques@ufpr.br

${ }^{3}$ Engenheira Florestal, Msc. em Engenharia Florestal - Universidade Federal do Paraná - Rua dos Funcionários, nº 1540 - 80.035-050 - Curitiba, PR carolbenghi@gmail.com

${ }^{4}$ Zoologista, Dr. - Staatliches Museum für Naturkunde Karlsruhe - Erbprinzenstrasse, 13 - 76133 - Karlsruhe, Alemanha - hubert.hoefer@smnk.de
}

Cerne, Lavras, v. 18, n. 1, p. 75-86, jan./mar. 2012 
A recuperação de uma floresta antropizada, em termos de diversidade e funcionalidade, depende de diversos fatores como histórico de uso da terra (CHAZDON, 2003; METZGER et al., 2009), tipo e extensão do distúrbio (CHAZDON, 2003), tamanho e conectividade dos fragmentos vegetacionais (METZGER et al., 2009), condições de áreas vizinhas (estado de preservação e grau de isolamento) (LAURENCE et al., 2002), proximidade das fontes de espécies, esporos e sementes (DUNN, 2004) e da diversidade funcional das comunidades de plantas e animais existentes nessas florestas secundárias (BIHN, 2008; WALKER, 1992). Nesse sentido, a ciclagem de nutrientes torna-se um aspecto muito importante para a recuperação dessas florestas.

O compartimento formado pela serapilheira-solo é o sítio onde ocorrem as etapas da decomposição da matéria orgânica e dessa ciclagem. Entretanto, não significa que os diversos fenômenos envolvidos nesse processo ocorram exclusivamente nessa estreita porção do ambiente, porém, é nesse compartimento onde se concentram os organismos responsáveis pela tarefa de fragmentar as cadeias carbônicas, elaboradas de maneira complexa pelos outros organismos autotróficos (CORREIA; ANDRADE, 1999). Representando uma ligação importante no ciclo orgânico de produção-decomposição, a serapilheira tem papel fundamental no funcionamento do ecossistema florestal (MEENTEMEYER et al., 1982). Dessa forma, seu estudo pode fornecer índices de produtividade da floresta, noções sobre taxas de decomposição e fenologia das espécies, além de permitir avaliar sua importância nos ciclos de nutrientes (CUNHA et al., 1993; DIDHAM, 1998; PROCTOR et al., 1983).

Com o intuito de contribuir para o entendimento da ecologia das florestas secundárias, neste estudo, objetivouse avaliar e quantificar a deposição de serapilheira em um trecho de Floresta Ombrófila Densa Submontana, no litoral do Paraná, em diferentes fases de sucessão secundária.

\section{MATERIAL E MÉTODOS}

\subsection{Localização e caracterização da área}

O presente trabalho foi realizado na Reserva Natural Rio Cachoeira (RNRC), situada na porção oeste-noroeste da Baía de Antonina (entre as coordenadas 2518'51'S e 48 41'45”'W), no município de Antonina, região litorânea do Estado do Paraná. A Reserva, pertencente à ONG Sociedade de Pesquisa em Vida Selvagem e Educação Ambiental (SPVS), possui uma área aproximada de 8.600 ha e faz parte da Zona de Conservação da Vida Silvestre da Área de Proteção Ambiental (APA) de Guaraqueçaba. É cercada ao norte e a leste pela Serra do Mar, ao sul pelo estuário dos rios Cachoeira e Faisqueira e a oeste por um trecho de planície, cruzado pelos rios Cacatu, do Nunes, Xaxim e Curitibaíba, que também encontra o sopé da referida Serra do Mar. A bacia hidrográfica está localizada na Baía de Antonina, cujo principal rio é o Cachoeira, que drena cerca de $700 \mathrm{~km}^{2}$, nos seus $48 \mathrm{~km}$ de extensão e banha parte da Reserva (SOCIEDADE DE PESQUISA EM VIDA SELVAGEM E EDUCAÇÃO AMBIENTAL - SPVS, 2000).

O tipo climático característico da área de estudo é o subtropical úmido mesotérmico (Cfa), com a temperatura média do mês mais frio inferior a $18^{\circ} \mathrm{C}$, porém superior a $-3^{\circ} \mathrm{C}$, e o mais quente apresentando temperatura média superior a $22{ }^{\circ} \mathrm{C}$. A região está sujeita a geadas pouco frequentes e há precipitações regulares todos os meses, sem apresentar estação seca definida (KOEPPEN, 1948). Em razão da expressão do seu relevo, com bruscas variações altimétricas, a temperatura média sobre esse ambiente diminui cerca de $0,6^{\circ} \mathrm{C}$ a cada $100 \mathrm{~m}$ de altitude. As precipitações nas encostas atingem valores entre 3300 a $3450 \mathrm{~mm}$ anuais, abastecendo uma rede fluvial que desempenha um papel fundamental no sistema de drenagem da planície (MAACK, 2002).

A vegetação foi classificada como "Floresta Ombrófila Densa Submontana”, situada entre 20 e 600 m de altitude (INSTITUTO BRASILEIRO DE GEOGRAFIA E ESTATÍSTICA - IBGE, 1992). A área que hoje pertence à RNRC e seus arredores foram intensivamente explorados no passado para extração de madeira, na base das montanhas, e deflorestada na planície para a implantação de pastagens e cultivos agrícolas. Portanto, a Reserva é caracterizada pela ocorrência de extensas áreas de florestas secundárias, sendo a maioria localizada na planície e sob influência do lençol freático ou na encosta de montanhas em solos originários de rochas ácidas do período neo précambriano (SPVS, 2000).

Para o desenvolvimento desta pesquisa foram selecionadas três áreas em diferentes fases sucessionais, denominadas fase inicial (INI), fase média (MED) e fase avançada (AVA). Cada fase sucessional foi representada por dez parcelas de $100 \mathrm{~m}^{2}$ cada, totalizando $1.000 \mathrm{~m}^{2}$. A distância aproximada entre as áreas é de $250 \mathrm{~m}$ entre as fases INI e MED e de $900 \mathrm{~m}$ entre estas e a fase AVA. A fase INI é uma formação pioneira, abandonada há aproximadamente 20 anos, após utilização pela pastagem.

Cerne, Lavras, v. 18, n. 1, p. 75-86, jan./mar. 2012 
A densidade de indivíduos arbóreos, medida no ano 2007, foi de 1890 ind.ha ${ }^{-1}$, dos quais 66\% correspondiam a uma espécie predominante, a Tibouchina pulchra (Cham.) Cogn. (jacatirão); a riqueza (número de espécies arbóreas por parcela) foi de nove espécies (em $1000 \mathrm{~m}^{2}$ ) e a área basal de 21,5 $\mathrm{m}^{2}$.ha-1 . A fase MED é uma formação intermediária, abandonada há aproximadamente 80 anos, após utilização para o cultivo da mandioca. A densidade de indivíduos arbóreos, medida no ano 2007, foi de 3006 ind.ha ${ }^{-1}$; a riqueza foi de 63 espécies arbóreas (em $1500 \mathrm{~m}^{2}$ ) e a área basal de $34,8 \mathrm{~m}^{2}$.ha-1 . A floresta onde situa-se a fase AVA sofreu a retirada de madeira com valor comercial há aproximadamente 120 anos. A densidade de indivíduos arbóreos, medida no ano 2007, foi de 1600 ind.ha $^{-1}$; a riqueza foi de 56 espécies arbóreas (em $1500 \mathrm{~m}^{2}$ ) e a área basal de 40,1 m².ha-1 (LIEBSCH et al., 2007). As florestas das fases INI e AVA estão assentadas sobre Cambissolo e, da fase MED, sobre Argissolo (FERRETI; BRITEZ, 2005).

\subsection{Coleta e processamento da serapilheira depositada}

A serapilheira foi coletada em coletores circulares de $0,25 \mathrm{~m}^{2}$, confeccionados com tubos de PVC e tela de náilon com malha de 2,0 $\mathrm{mm}^{2}$. Em cada parcela quadrada $(10 \mathrm{~m} \times 10 \mathrm{~m})$ foram instalados dois coletores, sendo estes alocados em extremidades opostas, na mesma diagonal da parcela. Na área total do estudo $\left(3000 \mathrm{~m}^{2}\right)$ foram instalados
60 coletores de serapilheira (20 em cada fase sucessional). As coletas foram realizadas a cada três semanas, de abril de 2004 a março de 2007, sendo que o material depositado nos 20 coletores de cada fase foi agrupado em uma única amostra. A cada coleta, a serapilheira foi acondicionada em sacos plásticos, secada ao ar livre e triada nas seguintes frações: Folhas das espécies selecionadas (composta pelas folhas de algumas espécies arbóreas selecionadas em cada fase pelo seu maior valor de importância e/ou maior ocorrência nos coletores de serapilheira, conforme Tabela 1); Fração Folhas (folhas das demais espécies que ocorreram no coletor e que não entraram na seleção anterior); Fração Ramos (ramos com até 2,0 cm de diâmetro); Fração Órgãos Reprodutivos (flores, frutos e sementes); Fração Miscelânea (todo material de origem animal e/ou vegetal que sobrou da triagem e não se enquadrou nas frações anteriores). Após triagem, a serapilheira foi secada em estufa a $60 \pm 5^{\circ} \mathrm{C}$ durante 72 horas e pesada. As quantidades depositadas nos coletores, em g.5 $\mathrm{m}^{-2}$, foram extrapoladas para kg.ha- ${ }^{-1}$.

\subsection{Análises estatísticas}

Para a realização das análises estatísticas, primeiramente foi verificado o cumprimento das condicionantes da análise de variância (variâncias homogêneas; erros aleatórios, independentes e normalmente distribuídos; médias e variâncias não correlacionadas).

Tabela 1 - Relação das espécies selecionadas nas fases inicial (INI), média (MED) e avançada (AVA) e seus respectivos valores de importância (VI), em porcentagem.

Table 1 - Tree species selected in the initial, medium and advanced sucessional stages and their respective importance values (IV), in percentage.

\begin{tabular}{|c|c|c|c|c|}
\hline Fase & Família & Espécie & Nome comum & VI $(\%)^{1}$ \\
\hline \multirow{2}{*}{ INI } & Melastomataceae & Tibouchina pulchra (Cham.) Cogn. & Jacatirão & 177,2 \\
\hline & Myrsinaceae & Myrsine coriacea (Sw.) R.Br. e Roem. \& Schult & Capororoca & 61,1 \\
\hline \multirow{4}{*}{ MED } & Rubiaceae & Bathysa meridionalis L.B. SM. \& Downs & Cajujão & 33,6 \\
\hline & Elaeocarpaceae & Sloanea guianensis (Aubl.) Benth. & Laranjeira-do-mato & 32,4 \\
\hline & Phyllanthaceae & Hyeronima alchorneoides Allemão & Licurana & 28,9 \\
\hline & Euphorbiaceae & Alchornea sp. & Tapiá & 13,7 \\
\hline \multirow{5}{*}{ AVA } & Myrtaceae & Marlieria tomentosa & Guapurunga & 45,6 \\
\hline & Elaeocarpaceae & Sloanea guianensis (Aubl.) Benth. & Laranjeira-do-mato & 18,2 \\
\hline & Phyllanthaceae & Hyeronima alchorneoides Allemão & Licurana & 7,6 \\
\hline & Urticaceae & Pourouma guianensis Aubl. & Embaubarana & 4,5 \\
\hline & Euphorbiaceae & Alchornea sp. & Tapiá & - \\
\hline
\end{tabular}

${ }^{1}$ Corresponde à soma da densidade, freqüência e dominância relativas.

Cerne, Lavras, v. 18, n. 1, p. 75-86, jan./mar. 2012 
Quando alguma dessas condicionantes não foi satisfeita no conjunto de dados em análise, procedeu-se à transformação dos mesmos (logaritmo, raiz quadrada, arco-seno). Quando os dados não cumpriram tais condicionantes mesmo após transformação, utilizou-se a análise não-paramétrica (Teste de Mann-Whitney). Na análise paramétrica, realizou-se a ANOVA, seguido de teste de comparação de médias (Teste de Fisher LSD 95\%). Todos os cálculos estatísticos foram realizados com o software STATISTICA 7.0.

\section{RESULTADOS E DISCUSSÃO}

Na Tabela 2, são apresentados os resultados da produção anual média de serapilheira e a contribuição percentual destas ao total produzido, sendo comparada a produção de serapilheira (frações e total) entre as fases sucessionais.

A produção da serapilheira total foi de 5201, 5399 e 5323 kg.ha-1 ano $^{-1}$ nas fases INI, MED e AVA, respectivamente, não diferindo estatisticamente entre as mesmas. As referências encontradas na literatura quanto ao padrão de deposição em florestas secundárias são controversas. Enquanto alguns trabalhos apontam para maiores produções de serapilheira em estádios sucessionais mais avançados (DANTAS; PHILLIPSON, 1989; MARTIUS et al., 2004; MCDONALD HEALY, 2000; PINTO et al., 2008; WERNECK et al., 2001), outros indicam que a deposição de serapilheira em estágios sucessionais iniciais e médios devem ser maiores do que em florestas maduras (EWEL, 1976; KELLMAN, 1970; LEITÃO FILHO, 1993; ONG et al., 1981). Cuevas e Medina (1986), estudando três tipos de floresta na região amazônica da Venezuela (Terra Firme, Caatinga e Bana) constataram que, apesar da grande diferença entre os três sítios, houve uma alta correlação entre as curvas de deposição de serapilheira, sugerindo que fatores comuns estariam regulando a deposição nos três ambientes. Leitão Filho (1993), em florestas secundárias atlânticas em

Tabela 2 - Produção anual média ${ }^{1}$ de serapilheira e contribuição percentual das frações da serapilheira produzida nas fases sucessionais inicial, média e avançada - comparação entre fases para cada fração da serapilheira e total.

Table 2 - Annual average litter fall production and percentage contribution of litter fall fractions in the initial, medium and advanced sucessional stages - comparison among sucessional stages for each fraction of litter fall and total.

\begin{tabular}{|c|c|c|c|c|c|c|c|c|c|}
\hline \multirow{2}{*}{ Fração da serapilheira } & \multicolumn{3}{|c|}{ Fase inicial } & \multicolumn{3}{|c|}{ Fase média } & \multicolumn{3}{|c|}{ Fase avançada } \\
\hline & Kg.ha $^{-1}$ ano $^{-1}$ & $\mathrm{EP}^{3}$ & $\%^{4}$ & Kg.ha $^{-1} \cdot$ ano $^{-1}$ & EP & $\%$ & Kg.ha $^{-1} \cdot$ ano $^{-1}$ & $\mathrm{EP}$ & $\%$ \\
\hline Ramos & $586 \mathrm{~A}^{2}$ & 72,7 & 11,3 & $588 \mathrm{~A}$ & 94,0 & 10,9 & $595 \mathrm{~A}$ & 73,2 & 11,2 \\
\hline Órgãos reprodutivos & $142 \mathrm{~A}$ & 24,1 & 2,7 & $186 \mathrm{~A}$ & 37,1 & 3,4 & $161 \mathrm{~A}$ & 44,9 & 3,0 \\
\hline Miscelânea & $487 \mathrm{~B}$ & 16,5 & 9,4 & $584 \mathrm{~B}$ & 74,0 & 10,8 & $957 \mathrm{~A}$ & 73,5 & 18,0 \\
\hline Folhas de Tibouchina pulchra & 2972 & 177,5 & 57,2 & - & & & - & - & - \\
\hline Folhas de Myrsine coriacea & 462 & 27,4 & 8,9 & - & & & - & - & - \\
\hline Folhas de Sloanea guianensis & - & & - & $454 \mathrm{~A}$ & 45,3 & 8,4 & $452 \mathrm{~A}$ & 24,5 & 8,5 \\
\hline Folhas de Hyeronima alchorneoides & - & & - & $336 \mathrm{~A}$ & 40,5 & 6,2 & $265 \mathrm{~A}$ & 12,9 & 5,0 \\
\hline Folhas de Alchornea sp. & - & & - & $148 \mathrm{~A}$ & 15,3 & 2,7 & $92 \mathrm{~B}$ & 5,8 & 1,7 \\
\hline Folhas de Bathysa meridionalis & - & & - & 218 & 18,2 & 4,0 & - & - & - \\
\hline Folhas de Pourouma guianensis & - & & - & - & - & - & 134 & 22,8 & 2,5 \\
\hline Folhas de Marlieria tomentosa & - & & - & - & - & - & 125 & 33,8 & 2,3 \\
\hline Folhas de outras espécies & $552 \mathrm{C}$ & 79,7 & 10,6 & $2887 \mathrm{~A}$ & 96,5 & 53,5 & 2543 B & 28,9 & 47,8 \\
\hline Folhas total & $3986 \mathrm{AB}$ & 157,9 & 76,6 & $4042 \mathrm{~A}$ & 140,4 & 74,9 & $3611 \mathrm{~B}$ & 43,7 & 67,8 \\
\hline Serapilheira total & $5201 \mathrm{~A}$ & 150,4 & 100 & $5399 \mathrm{~A}$ & 289,9 & 100 & $5323 \mathrm{~A}$ & 129,2 & 100 \\
\hline
\end{tabular}

${ }^{1}$ Média dos 4 anos de estudo (2004 a 2007).

${ }^{2}$ Valores em linha seguidos da mesma letra maiúscula não diferem estatisticamente pelo teste de Fisher $(P<0,05)$ - comparação entre fases sucessionais.

${ }^{3} \mathrm{EP}=$ erro padrão da média.

${ }^{4}$ Contribuição percentual das frações da serapilheira.

Cerne, Lavras, v. 18, n. 1, p. 75-86, jan./mar. 2012 
Cubatão (SP), em diferentes fases sucessionais, também encontrou padrão qualitativo de deposição semelhante entre as áreas. Bencke e Morellato (2002), estudando a fenologia de espécies arbóreas na floresta atlântica em Ubatuba (SP), observaram que populações localizadas em tipos florestais diferentes, porém próximas entre si e sujeitas às mesmas condições climáticas mantêm, em geral, o mesmo comportamento fenológico. Barlow et al. (2007), em estudo de produção e decomposição de serapilheira em florestas primárias, secundárias e plantadas na Amazônia brasileira, não encontraram diferença na produção de serapilheira entre florestas secundárias e primárias.

No presente estudo, a quantidade de serapilheira depositada foi semelhante nas três fases sucessionais. É provável que a condição climática local, semelhante nas três áreas, decorrente da proximidade entre si, tenha sido um dos fatores responsáveis pela deposição não ter diferido significativamente entre as mesmas. Outra questão relevante é a estrutura da floresta. Conforme estudo conduzido por Liebsch et al. (2007) nas mesmas áreas de estudo, as fases MED e AVA apresentaram características quantitativas (área basal, altura, volume) e qualitativas (riqueza, diversidade) semelhantes entre si e maiores que as observadas na fase INI. Por esse fato, seria de se esperar que a quantidade de serapilheira produzida na fase INI fosse diferente da quantidade produzida nas fases MED e AVA, o que não aconteceu. Esse resultado foi observado, provavelmente, pela grande influência da espécie $T$. pulchra na fase INI, cuja serapilheira foliar representou mais da metade (57\%) da serapilheira total. Além disso, o fato de a floresta na fase INI possuir menor área basal, menor volume de madeira, menor riqueza e diversidade de espécies vegetais, sub-bosque pouco desenvolvido, dossel descontínuo, entre outras características, pode ter contribuído para uma maior incidência luminosa e, consequentemente, maior produção de fitomassa.

A produção da fração folhas total foi de 3986, 4042 e 3611 kg.ha- ${ }^{-1}$.ano ${ }^{-1}$ nas fases INI, MED e AVA, respectivamente. Essa produção somente diferiu estatisticamente entre as fases MED e AVA. A contribuição das folhas à serapilheira total $(77,75$ e $68 \%$ nas fases INI, MED e AVA, respectivamente) está de acordo com a maioria dos estudos de produção de serapilheira em florestas tropicais, onde são relatadas maiores contribuições destas ao total de serapilheira produzida. Essa porcentagem, normalmente situa-se acima dos 60-70\% (CUEVAS; MEDINA, 1986; CUNHA et al., 1993; CUSTÓDIO FILHO et al., 1996; LUIZÃO; SCHUBART, 1986;
MACHADO et al., 2008; MARTIUS et al., 2004; MORAES et al., 1999; PINTO; MARQUES, 2003; ROCHA, 2006; SCHEER, 2006; SILVA et al., 2009; VARJABEDIAN; PAGANO, 1988; VOGEL et al., 2007). A diminuição na porcentagem de folhas (de 77 para 68\%), com o avanço da fase sucessional, já foi observada por outros autores em florestas tropicais secundárias em diferentes fases de sucessão (CUNHA, 1997; KÖHLER et al., 2008; PEZZATO; WISNIEWSKI, 2006; PINTO; MARQUES, 2003; ROCHA, 2006; TOLEDO et al., 2002) e pode estar associada a uma maior produção de serapilheira foliar pelas espécies pioneiras presentes nas florestas secundárias em processo inicial de regeneração (MARTINS; RODRIGUES, 1999). Luizão e Schubart (1986), investigando a produção de serapilheira em floresta de terra firme da Amazônia Central, encontraram maior porcentagem de folhas na capoeira jovem em relação às florestas originais de platô e baixio. A maior porcentagem de folhas depositadas na capoeira foi justificada pelos autores como decorrente do crescimento acelerado em que se encontrava a vegetação, para o qual necessitava de uma alta taxa fotossintética, conseguida por meio de uma grande biomassa de folhas que eram rapidamente substituídas. Por outro lado, esta maior proporção de folhas nas florestas iniciais, comparativamente às florestas mais velhas, reflete a estrutura da vegetação e a diversidade biológica, diferentes em florestas com diferentes idades. Nas florestas mais novas, o tipo de material que cai das árvores é principalmente de natureza foliar, pela própria estrutura das plantas (estacionalmente, flores e frutos podem ser significativos). À medida que a floresta envelhece, além da renovação das folhas, outros materiais (cascas, ramos de maiores dimensões, epífitas, etc) também passam a cair nos coletores, sendo que boa parte deste material não se encontrava presente nas florestas mais jovens, o que explicaria a redução na proporção de folhas. Adicionalmente, nas florestas mais velhas, a presença de insetos e pássaros pode ser mais expressiva, contribuindo para a deposição de materiais de menores dimensões, como foi observado na fração miscelânea deste estudo.

Com relação à contribuição percentual das folhas das espécies selecionadas na fase INI, mais da metade da serapilheira total (57\%) constituiu-se de folhas da Tibouchina pulchra. O predomínio das folhas dessa espécie sobre as demais foi consequência do maior valor de importância da mesma (177\%). Na fase MED, esta espécie está praticamente ausente no ambiente e um número maior de outras espécies se estabeleceu. Enquanto na fase INI 75\% da serapilheira foliar era composta pela T. pulchra e apenas

Cerne, Lavras, v. 18, n. 1, p. 75-86, jan./mar. 2012 
25\% por outras espécies, na fase MED um número maior de outras espécies passou a contribuir com a deposição de serapilheira. Dentre essas espécies foram selecionadas quatro, pelo seu maior valor de importância e ocorrência nos coletores de serapilheira. Essas espécies foram Sloanea guianensis, Hyeronima alchorneoides, Bathysa meridionalis e Alchornea sp., cuja participação na serapilheira total foi de 8,4, 6,2, 4,0 e 2,7\%, respectivamente. A contribuição percentual das folhas das outras espécies (53,5\%) foi cinco vezes superior à contribuição das folhas das outras espécies da fase INI (10,6\%). Na fase AVA, as folhas das espécies S. guianensis, H. alchorneoides e Alchornea sp. continuaram a ter expressão na serapilheira depositada e duas outras espécies, Pourouma guianensis e Marlieria tomentosa, foram incluídas na triagem. A contribuição das folhas dessas espécies à serapilheira total foi de 8,5, 5,0, 1,7, 2,5 e 2,3\%, respectivamente. Enquanto a contribuição das folhas de T. pulchra à serapilheira total foi de $57,2 \%$, a maior contribuição das demais espécies estudadas não ultrapassou os 9\%. Essa maior deposição das espécies pioneiras está relacionada a um retorno maior de folhas e a uma vida média mais curta das mesmas, contribuindo para aumentar a fertilidade das camadas superiores do solo, especialmente na sucessão secundária (CHABOT; HICKS, 1982; RUIZJAÉN; AIDE, 2005 citados por BENVENUTI-FERREIRA et al., 2009). Das espécies selecionadas nas fases MED e AVA, a S. guianensis foi a que mais contribuiu na deposição de folhas, sendo essa contribuição similar entre as fases onde ela ocorreu (454 e $452 \mathrm{~kg} \cdot \mathrm{ha}^{-1}$, respectivamente). Apesar de haver 63 indivíduos de $S$. guianensis na fase MED contra apenas quatro na fase AVA (LIEBSCH et al., 2007), os indivíduos desta última possuem diâmetro bastante superior, resultando numa área basal próxima entre as duas fases $\left(4,03 \mathrm{~m}^{2}\right.$.ha-1 na fase MED e 5,58 $\mathrm{m}^{2}$.ha- ${ }^{-1}$ na fase AVA). Portanto, a deposição de quantidades similares de folhas por essa espécie, nas duas fases sugere que diâmetro de fuste e diâmetro de copa estejam positivamente correlacionados, ou seja, como os indivíduos na fase AVA possuem maior diâmetro do fuste, provavelmente suas copas sejam maiores também e, por isso, depositem mais folhas do que os indivíduos de S. guianensis na fase MED, cujos diâmetros são menores. Entretanto, essa é apenas uma hipótese, pois as características da copa (diâmetro, área de projeção, forma, etc) dependem de diversos fatores do sítio como densidade, competição entre copas, posição na estrutura vertical da floresta, entre outros, os quais não foram mensurados. Para as espécies H. alchorneoides e Alchornea sp. observou-se uma deposição de folhas um pouco maior na fase MED em relação à AVA, apesar da análise estatística não haver detectado diferença entre fases para as folhas de H. alchorneoides.

Adeposição de ramos foi estimada em 586, 588 e 595 kg.ha ${ }^{-1}$.ano ${ }^{-1}$, nas fases INI, MED e AVA, respectivamente, não havendo diferença estatística significativa entre as fases. A deposição de órgãos reprodutivos também não diferiu entre as fases INI, MED, AVA (142, 186 e 161 $\mathrm{kg} \cdot \mathrm{ha}^{-1}$. ano ${ }^{-1}$, respectivamente). Como não houve diferença entre as três fases sucessionais, a contribuição percentual média de ramos e órgãos reprodutivos à serapilheira total foi de 11 e 3\%, respectivamente. Já, a deposição da miscelânea foi maior na fase AVA $\left(957 \mathrm{~kg} \cdot \mathrm{ha}^{-1} \cdot\right.$ ano $\left.^{-1}\right)$ e não diferiu entre as fases INI e MED. A contribuição percentual da fração miscelânea foi de 9, 11 e 18\% nas fases INI, MED e AVA, respectivamente. A comparação desses resultados com outros encontrados na literatura torna-se complexa, em razão de uma grande variação natural na deposição dessas frações e às formas de amostragem e triagem utilizadas. Alguns autores computaram os pesos dos ramos juntamente com os órgãos reprodutivos (PINTO; MARQUES, 2003; ROCHA, 2006), outros separaram os órgãos reprodutivos em fração flores e fração frutos (TOLEDO et al., 2002), outros triaram a serapilheira somente em duas frações, foliar e lenhosa (GAMA-RODRIGUES; BARROS, 2002; LUIZÃO; SCHUBART, 1986; WERNECK et al., 2001) ou, ainda, não fizeram distinção entre os elementos reprodutivos e a miscelânea (CUSTÓDIO FILHO et al., 1996; FIGUEIREDO FILHO et al., 2003; KÖNIG et al., 2002; LEITÃO FILHO, 1993; MORELLATO, 1992; PAGANO, 1989; PEZZATO; WISNIEWSKI, 2006; PINTO et al., 2008; SILVA, 1984). Diante do exposto, as comparações devem ser cautelosas, levando-se em conta essas possíveis variações. Nos trabalhos realizados na Floresta Ombrófila Densa Submontana, a contribuição dos ramos variou de 12 a $25 \%$ e a de elementos reprodutivos mais miscelânea de 0,3 a $15 \%$.

Comparando-se a produção das frações da serapilheira dentro de cada fase sucessional (Tabela 3), observou-se que, na fase INI, a maior produção foi de folhas da espécie T. pulchra, seguido da fração ramos, folhas de outras espécies, fração miscelânea e folhas da espécie $M$. coriacea, com uma produção anual em torno de $500 \mathrm{~kg} \cdot \mathrm{ha}^{-1}$ (sem diferença estatística significativa entre as frações) e, por último, com a menor produção de serapilheira, a fração órgãos reprodutivos.

Na fase MED, a maior produção de serapilheira foi de folhas das outras espécies, seguido das frações ramos, miscelânea e folhas da espécie $S$. guianensis

Cerne, Lavras, v. 18, n. 1, p. 75-86, jan./mar. 2012 
(em torno de $540 \mathrm{~kg} \cdot \mathrm{ha}^{-1}$, sem diferença estatística significativa). A menor produção foi de folhas das espécies
H. alchorneoides, B. meridionalis e Alchornea sp., e da fração órgãos reprodutivos.

Tabela 3 - Produção anual média de serapilheira (frações e total) nas fases sucessionais inicial, média e avançada - comparação entre frações em cada fase sucessional.

Table 3 - Annual average litter fall production (fractions and total) in the initial, medium and advanced sucessional stages comparison among litter fall fractions in each sucessional stage.

\begin{tabular}{|c|c|c|c|c|}
\hline Fase & Fração da serapilheira & $\begin{array}{c}\text { Deposição }^{1} \\
\left(\mathrm{~kg} \cdot \mathrm{ha}^{-1} \cdot \text { ano }^{-1}\right)\end{array}$ & $\mathrm{EP}^{4}$ & $\mathrm{CV}^{5}(\%)$ \\
\hline \multirow{8}{*}{ Inicial } & Ramos & $586 \mathrm{~B}^{2}$ & 72,7 & 24,8 \\
\hline & Órgãos reprodutivos & $142 \mathrm{C}$ & 24,1 & 34,0 \\
\hline & Miscelânea & $487 \mathrm{~B}$ & 16,5 & 6,8 \\
\hline & Tibouchina pulchra & 2972 A & 177,5 & 11,9 \\
\hline & Myrsine coriacea & $462 \mathrm{~B}$ & 27,4 & 11,9 \\
\hline & Outras folhas & 552 B & 79,7 & 28,9 \\
\hline & Folhas total & 3986 & 157,9 & 7,9 \\
\hline & Serapilheira total & 5201 & 150,4 & 5,8 \\
\hline \multirow{10}{*}{ Média } & Ramos & $588 \mathrm{~B}$ & 94,0 & 32,0 \\
\hline & Órgãos reprodutivos & $186 \mathrm{D}$ & 37,1 & 40,0 \\
\hline & Miscelânea & 584 B & 74,0 & 25,3 \\
\hline & Sloanea guianensis & $454 \mathrm{BC}$ & 45,3 & 20,0 \\
\hline & Hyeronima alchorneoides & $336 \mathrm{C}$ & 40,5 & 24,1 \\
\hline & Alchornea sp. & $148 \mathrm{D}$ & 15,3 & 20,6 \\
\hline & Bathysa meridionalis & $218 \mathrm{D}$ & 18,2 & 16,7 \\
\hline & Outras folhas & $2887 \mathrm{~A}$ & 96,5 & 6,7 \\
\hline & Folhas total & 4042 & 140,4 & 6,9 \\
\hline & Serapilheira total & 5399 & 298,9 & 11,1 \\
\hline \multirow{11}{*}{ Avançada } & Ramos & $595 C^{3}$ & 73,2 & 24,6 \\
\hline & Órgãos reprodutivos & $161 \mathrm{DE}$ & 44,9 & 56,0 \\
\hline & Miscelânea & $957 \mathrm{~B}$ & 73,5 & 15,4 \\
\hline & Sloanea guianensis & $452 \mathrm{C}$ & 24,5 & 10,8 \\
\hline & Hyeronima alchorneoides & $265 \mathrm{D}$ & 12,9 & 9,7 \\
\hline & Alchornea sp. & $92 \mathrm{E}$ & 5,8 & 12,7 \\
\hline & Pourouma guianensis & $134 \mathrm{E}$ & 22,8 & 33,9 \\
\hline & Marlieria tomentosa & $125 \mathrm{E}$ & 33,8 & 54,1 \\
\hline & Outras folhas & 2543 A & 28,9 & 2,3 \\
\hline & Folhas total & 3611 & 43,7 & 2,4 \\
\hline & Serapilheira total & 5323 & 129,2 & 4,9 \\
\hline
\end{tabular}

${ }^{1}$ Média dos 4 anos de estudo (2004 a 2007).

${ }^{2}$ Valores em coluna seguidos da mesma letra maiúscula não diferem estatisticamente pelo teste de Fisher $(P<0,05)$. Os dados das fases INI e MED foram transformados por logaritmo.

${ }^{3}$ Valores em coluna seguidos da mesma letra maiúscula não diferem estatisticamente pelo teste de Mann-Whitney. Os dados da fase AVA foram analisados por análise não-paramétrica.

${ }^{4} \mathrm{EP}=$ erro padrão da média

${ }^{5} \mathrm{CV}=$ coeficiente de variação

Cerne, Lavras, v. 18, n. 1, p. 75-86, jan./mar. 2012 
Na fase AVA, da mesma forma como ocorrido na fase MED, a maior produção de serapilheira foi de folhas das outras espécies, seguido da fração miscelânea, fração ramos, folhas da espécie $S$. guianensis, folhas da espécie H. alchorneoides e, com menor produção, fração órgãos reprodutivos, folhas das espécies $P$. guianensis, M. tomentosa e Alchornea sp. Portanto, nas três fases sucessionais, a maior produção foi de folhas e a menor de órgãos reprodutivos.

A quantidade de serapilheira depositada sobre o solo, em florestas tropicais em geral, varia de 4 a 25 t.ha${ }^{1}$.ano ${ }^{-1}$ (GOLLEY et al., 1978). Na Floresta Ombrófila Densa Atlântica, os estudos já realizados estimaram quantidades variando de 1,8 a 9,5 t.ha- ${ }^{-1}$.ano ${ }^{-1}$, porém, na maioria, esses valores estão entre 5 e 8 t.ha ${ }^{-1}$.ano ${ }^{-1}$. Alguns trabalhos encontrados na literatura mostraram valores de deposição de serapilheira em Floresta Ombrófila Densa Amazônica (várzea, igapó e terra firme) similares à deposição da Floresta Ombrófila Mista (entre 6,1 e 9,4 t.ha- ${ }^{1} \cdot$ ano $^{-1}$ ). Já, para as florestas estacionais (deciduais e semideciduais), a quantidade de estudos realizados é maior e mostra uma maior amplitude nas quantidades depositadas (2,2 a 11,7 t.ha ${ }^{-1} \cdot$ ano $\left.^{-1}\right)$. As quantidades estimadas no presente estudo, 5,3 t.ha ${ }^{-1}$. ano ${ }^{-1} \mathrm{em}$ média, estão próximas às encontradas em outras tipologias florestais brasileiras (Tabela 4).

Tabela 4 - Deposição anual de serapilheira $\left({\mathrm{t} . h a^{-1} \text {.ano }}^{-1}\right)$ em diferentes tipologias florestais brasileiras.

Table 4 - Annual average litter fall production $\left(\right.$ t.ha $^{-1}$.year ${ }^{-1}$ ) of different Brazilian forest typologies.

\begin{tabular}{|c|c|c|c|c|}
\hline Vegetação & Local & Solo & $\begin{array}{l}\text { Serapilheira } \\
\text { depositada } \\
\left(\text { t.ha }^{-1} \cdot \text {.no }^{-1}\right)\end{array}$ & Referência \\
\hline $\begin{array}{l}\text { Floresta Ombrófila Densa Submontana } \\
\text { (formações secundárias) }\end{array}$ & Cubatão, SP & $\begin{array}{c}\text { Argissolo, } \\
\text { Cambissolo, } \\
\text { Neossolo }\end{array}$ & 4,5 a 5,7 & Leitão Filho (1993) \\
\hline Restinga inundável & Paranaguá, PR & Espodossolo & 5,2 & Britez (1994) \\
\hline Restinga & Paranaguá, PR & Espodossolo & 5,1 & Pires et al. (2006) \\
\hline $\begin{array}{l}\text { Floresta Ombrófila Densa das Terras } \\
\text { Baixas (formação secundária inicial) }\end{array}$ & Paranaguá, PR & Espodossolo & 5,3 & Rocha (2006) \\
\hline Floresta Estacional Semidecidual & Parobé, RS & Cambissolo & 5,5 & Mello (1995) \\
\hline $\begin{array}{l}\text { Floresta Estacional Semidecidual } \\
\text { (formação secundária inicial) }\end{array}$ & Ouro Preto, MG & Neossolo & 5,1 & Werneck et al. (2001) \\
\hline Cerrado & Sudeste do Brasil & Latossolo & 5,6 & Valenti et al. (2008) \\
\hline
\end{tabular}

\section{CONCLUSÕES}

Os resultados deste trabalho mostram que nas fases inicial e média da sucessão florestal, a produção de serapilheira pode ser equivalente ou mesmo superior àquela de fases sucessionais avançadas, em termos quantitativos. Entretanto, qualitativamente, essa produção fica restrita à contribuição de poucas espécies florestais, diferentemente do que acontece nas florestas mais velhas, onde a diversidade vegetal é maior. Quanto à contribuição das diferentes espécies, fica evidente o papel das espécies pioneiras na fase inicial da sucessão, no sentido de preparar o ambiente para as espécies subsequentes, por meio de uma maior produção e renovação da fitomassa. A redução na produção de serapilheira foliar e aumento da produção de miscelânea ao longo do processo sucessional revelam mudanças nas características estruturais da floresta e na diversidade biológica, inerentes aos processos sucessionais. As folhas, independentemente da fase sucessional, contribuem com o maior aporte de fitomassa ao solo florestal.

\section{AGRADECIMENTOS}

O presente estudo foi desenvolvido dentro do Projeto SOLOBIOMA, um projeto de cooperação BrasilAlemanha do Programa Mata Atlântica, financiado pelo Ministério de Educação e Ciência da Alemanha (BMBF) e pelo Conselho Nacional de Desenvolvimento Científico e Tecnológico (CNPq). A Sociedade de Pesquisa em Vida Selvagem e Educação Ambiental (SPVS) fez a cessão da

Cerne, Lavras, v. 18, n. 1, p. 75-86, jan./mar. 2012 
área de estudo e deu apoio logístico para a realização dos trabalhos de campo e a Universidade Federal do Paraná (UFPR) forneceu a infraestrutura de laboratórios para o processamento e análises do material coletado.

\section{REFERÊNCIAS}

BARLOW, J.; GARDNER, T. A.; FERREIRA, L. V.; PERES, C. A. Litter fall and decomposition in primary, secondary and plantation forests in the Brazilian Amazon. Forest Ecology and Management, Victoria, v. 246, p. 91-97, 2007.

BENCKE, C. S. C.; MORELLATO, L. P. C. Estudo comparativo da fenologia de nove espécies arbóreas em três tipos de floresta atlântica no sudeste do Brasil. Revista Brasileira de Botânica, São Paulo, v. 25, n. 2, p. 237-248, 2002.

BENVENUTTI-FERREIRA, G.; COELHO, G. C.; SCHIRMER, J.; LUCCHESE, O. A. Dendrometry and litterfall of neotropical pioneer and early secondary tree species. Biota Neotropica, São Paulo, v. 9, n. 1, p. 65-71, 2009.

BIHN, J. The recovery of ant communities in regenerating tropical forests, Marburg/Lahn. 2008. 97 p. Thesis (Ph.D. in Nature Sciences) - University of Marburg, Marburg, 2008.

BRITEZ, R. M. Ciclagem de nutrientes minerais em duas florestas da planície litorânea da Ilha do Mel, Paranaguá. 1994. 272 f. Dissertação (Mestrado em Ciência do Solo) Universidade Federal do Paraná, Curitiba, 1994.

CHAZDON, R. L. Tropical forest recovery: legacies of human impact and natural disturbances. Perspectives in Plant Ecology Evolution and Systematics, Zurich, v. 6, p. 51-71, 2003.

CORREIA, M. E. F.; ANDRADE, A. G. Formação de serapilheira e ciclagem de nutrientes. In: SANTOS, G. A.; CAMARGO, F. A. O. Fundamentos da matéria orgânica do solo: ecossistemas tropicais e subtropicais. Porto Alegre: Gênesis, 1999. p. 197-225.

CUEVAS, E.; MEDINA, E. Nutrient dynamics within Amazonian forest ecosystems: I., nutrient flux in fine litter fall and efficiency of nutrient utilization. Oecologia, Marburg, v. 68, p. 466-472, 1986.

CUNHA, G. C. Aspectos da ciclagem de nutrientes em diferentes fases sucessionais de uma floresta estacional do
Rio Grande do Sul. 1997. 86 f. Dissertação (Mestrado em Ciências Florestais) - Escola Superior de Agricultura "Luiz de Queiroz”, Piracicaba, 1997.

CUNHA, G. C.; GRENDENE, L. A.; DURLO, M. A.; BRESSAN, D. A. Nutrient cycling in a seasonal deciduous forest with special respect to the mineral content produced by the litter fall. Ciência Florestal, Santa Maria, v. 3, n. 1, p. 36-64, 1993.

CUSTÓDIO FILHO, A.; FRANCO, G. A. D. C.; POGGIANI, F.; DIAS, A. C. Produção de serapilheira e o retorno de macronutrientes em Floresta Pluvial Atlântica: estação biológica de Boracéia, São Paulo, Brasil. Revista do Instituto Florestal, São Paulo, v. 8, n. 1, p. 1-16, 1996.

DANTAS, M.; PHILLIPSON, J. Litterfall and litter nutrient content in primary and secondary Amazonian "terra firme" rain forest. Journal of Tropical Ecology, Winchelsea, v. 5, n. 1, p. 27-36, 1989.

DIDHAM, R. K. Altered leaf-litter decomposition rates in tropical forest fragments. Oecologia, Marburg, v. 116, p. 397406, 1998.

DUNN, R. R. Recovery of faunal communities during tropical forest regeneration. Conservation Biology, Santa Barbara, v. 18, p. 302-309, 2004.

EWEL, J. J. Litter fall and leaf decomposition in a tropical forest sucession in eastern Guatemela. Journal of Ecology, London, v. 64, p. 293-308, 1976.

FERRETI, A. R.; BRITEZ, R. M. A restauração da floresta Atlântica no litoral do estado do Paraná: os trabalhos da SPVS. In: GALVÃO, A. P. M.; SILVA, P. (Ed.). Restauração Florestal: fundamentos e estudos de caso. Colombo: Embrapa, 2005. p. 87-102.

FIGUEIREDO FILHO, A.; MORAES, G. F.; SCHAAF, L. B.; FIGUEIREDO, D. J. Avaliação estacional da deposição de serapilheira em uma Floresta Ombrófila Mista localizada no sul do Estado do Paraná. Ciência Florestal, Santa Maria, v. 13, n. 1, p. 11-18, 2003.

GAMA-RODRIGUES, A. C.; BARROS, N. F. Ciclagem de nutrientes em floresta natural e em plantios de eucalipto e de dandá no sudeste da Bahia, Brasil. Revista Árvore, Viçosa, v. 26, n. 2, p. 193-207, mar./abr. 2002.

Cerne, Lavras, v. 18, n. 1, p. 75-86, jan./mar. 2012 
GOLLEY, F. B.; MCGINNIS, J. T.; CLEMENTS, R. G.; CHILD, G. I.; DUEVER, M. J. Ciclagem de minerais em um ecossistema de floresta tropical úmida. São Paulo: EDUSP, 1978. $256 \mathrm{p}$.

\section{INSTITUTO BRASILEIRO DE GEOGRAFIA E} ESTATÍSTICA. Manual técnico da vegetação brasileira. Rio de Janeiro: DEDIT/CDDI, 1992. 92 p. (Série manuais técnicos em geociências).

KELLMAN, M. C. Secondary plant succession in tropical montane Mindanao. Canberra: Australian National University, 1970. 174 p.

KÖHLER, L.; HÖLSCHER, D.; LEUSCHNER, C. High litterfall in old-growth and secondary upper montane forest of Costa Rica. Plant Ecology, Perth, v. 199, p. 163-173, 2008.

KÖNIG, F. G.; SCHUMACHER, M. V.; BRUN, E. J.; SELING, I. Avaliação da sazonalidade da produção de serapilheira numa Floresta Estacional Decidual no município de Santa Maria, RS. Revista Árvore, Viçosa, v. 26, n. 4, p. 641-648, jul./ago. 2002.

LAURENCE, W. F.; LOVEJOY, T. E.; VASCONCELOS, H. L.; BRUNA, E. M.; DIDHAM, R. K.; STOUFFER, P. C.; GASCON, C.; BIERREGAARD, R. O.; LAURANCE, S. G.; SAMPAIO, E. Ecosystem decay of Amazonian forest fragments: a 22-year investigation. Conservation Biology, Santa Barbara, v. 16, p. 605-618, 2002.

LEITÃO FILHO, H. F. (Coord.). Ecologia da Mata Atlântica em Cubatão. São Paulo: Unesp; Campinas: Unicamp, 1993. $184 \mathrm{p}$.

LIEBSCH, D.; GOLDENBERG, R.; MARQUES, M. C. M. Florística e estrutura de comunidades vegetais em uma cronosequência de Floresta Atlântica no Estado do Paraná, Brasil. Acta Botanica Brasilica, Feira de Santana, v. 21, n. 4, p. 983-992, 2007.

LUIZÃO, F. J.; SCHUBART, H. O. R. Produção e decomposição de liteira em Floresta de Terra Firme da Amazônia Central. Acta Limnologica Brasiliensia, Rio Claro, v. 1, p. 575-600, 1986.

MAACK, R. Geografia física do Estado do Paraná. 3. ed. Curitiba: UFPR, 2002. 440 p.

Cerne, Lavras, v. 18, n. 1, p. 75-86, jan./mar. 2012
MACHADO, M. R.; RODRIGUES, F. C. M. P.; PEREIRA, M. G. Produção de serapilheira como bioindicador de recuperação em plantio adensado de revegetação. Revista Árvore, Viçosa, v. 32, n. 1, p. 143-151, jan./fev. 2008.

MARTINS, S. V.; RODRIGUES, R. R. Produção de serapilheira em clareiras de uma floresta estacional semidecidual no município de Campinas, SP. Revista Brasileira de Botânica, São Paulo, v. 22, p. 405-412, 1999.

MARTIUS, C.; HÖFER, H.; GARCIA, M. V. B.; RÖMBKE, J.; HANAGARTH, W. Litter fall, litter stocks and decomposition rates in rainforest and agroforestry sites in central Amazônia. Nutrient Cycling in Agroecosystems, Bohn, v. 68, p. 137-154, 2004.

MCDONALD, M. A.; HEALEY, J. R. Nutrient cycling in secondary forest in the Blue Mountains of Jamaica. Forest Ecology and Management, Victoria, v. 139, p. 257-278, 2000.

MEENTEMEYER, V.; BOX, E. O.; THOMPSON, R. World patterns and amounts of terrestrial plant litter production. BioScience, Uberlândia, v. 32, n. 2, p. 125-128, 1982.

MELLO, R. S. P. Produção de serapilheira e aspectos da ciclagem de nutrientes em dois tipos florestais adjacentes no Rio Grande do Sul. 1995. 135 f. Dissertação (Mestrado em Ecologia) - Universidade Federal do Rio Grande do Sul, Porto Alegre, 1995.

METZGER, J. P. Conservation issues in the Brazilian Atlantic forest. Biological Conservation, Boston, v. 142, p. 11381140, 2009.

METZGER, J. P.; MARTENSEN, A. C.; DIXO, M.; BERNACCI, L. C.; RIBEIRO, M. C.; TEIXEIRA, A. M. G.; PARDINI, R. Time-lag in biological responses to landscape changes in a highly dynamic Atlantic forest region. Biological Conservation, Boston, v. 142, p. 1166-1177, 2009.

MORAES, R. M.; DELITTI, W. B.; STRUFFALDI-DEVUONO, Y. Litterfall and litter nutrient content in two Brazilian Tropical Forest. Revista Brasileira de Botânica, São Paulo, v. 22, n. 1, p. 9-16, 1999.

MORELLATO, L. P. C. Nutrient cycling in two south-east Brazilian forests: I., litterfall and litter standing crop. Journal of Tropical Ecology, Winchelsea, v. 8, p. 205-215, 1992. 
ONG, J. E.; GONK, W. K.; WONG, C. H. Productivity of a managed mangrove forest in West Malaysia. In: INTERNATIONAL CONFERENCE ON TRENDS IN APPLIED BIOLOGY IN SOUTH EAST ASIA, 1981, Penang. Proceedings... Penang: University Sains Malaysis, 1981. p. 274-284.

PAGANO, S. N. Produção de folhedo em mata mesófila semidecídua no município de Rio Claro, SP. Revista Brasileira de Biologia, São Carlos, v. 49, n. 3, p. 633-639, 1989.

PEZZATO, A. W.; WISNIEWSKI, C. Produção de serapilheira em diferentes seres sucessionais da Floresta Estacional Semidecidual no oeste do Paraná. Revista Floresta, Curitiba, v. 36, n. 1, p. 111-120, 2006.

PINTO, C. B.; MARQUES, R. Aporte de nutrientes por frações da serapilheira em sucessão ecológica de um ecossistema da Floresta Atlântica. Revista Floresta, Curitiba, v. 33, n. 3, p. 257-264, 2003.

PINTO, S. I. C.; MARTINS, S. V.; BARROS, N. F.; DIAS, H. C. T. Produção de serapilheira em dois estádios sucessionais de Floresta Estacional Semidecidual na Reserva Mata do Paraíso, em Viçosa, MG. Revista Árvore, Viçosa, v. 32, n. 3, p. 545-556, maio/jun. 2008.

PIRES, L. A.; BRITEZ, R. M. de; MARTEL, G.; PAGANO, S. N. Produção e acúmulo e decomposição da serapilheira em uma restinga da Ilha do Mel, Paranaguá, PR, Brasil. Acta Botanica Brasilica, Feira de Santana, v. 20, n. 1, p. 173-184, 2006.

PROCTOR, J.; ANDERSON, J. M.; FOGDEN, S. C. L.; WALLACK, H. W. Ecological studies in four contrasting lowland rain forests in Gulung Mulu National Park, Sarawak. Journal of Ecology, London, v. 71, p. 262-283, 1983.

RIBEIRO, M. C.; METZGER, J. P.; MARTENSEN, A. C.; PONZONI, F. J.; HIROTA, M. M. The Brazilian Atlantic Forest: how is left, and how is the remaining forest disturbed?: implications for conservation. Biological Conservation, Boston, v. 142, p. 1141-1153, 2009.

ROCHA, A. A. Deposição de fitomassa e nutrientes, acumulação e decomposição de serapilheira em três tipologias da Floresta Atlântica, Paranaguá, PR. 2006. 98

p. Dissertação (Mestrado em Ciência do Solo) - Universidade Federal do Paraná, Curitiba, 2006.
SCHEER, M. B. Ciclagem de nutrientes em um trecho de Floresta Ombrófila Densa Aluvial em regeneração Guaraqueçaba, PR. 2006. 155 p. Dissertação (Mestrado em Ciências Florestais) - Universidade Federal do Paraná, Curitiba, 2006.

SILVA, C. J. da; LOBO, F. A.; BLEICH, M. E.; SANCHES, L. Contribuição de folhas na formação da serapilheira e do retorno de nutrientes em floresta de transição no norte de Mato Grosso. Acta Amazonica, Manaus, v. 39, n. 3, p. 591-600, 2009.

SILVA, M. F. F. Produção anual de serapilheira e seu conteúdo mineralógico em mata tropical de terra firme, Tucuruí, PA.

Boletim do Museu Paraense Emílio Goeldi, Belém, v. 1, n. 1/2, p. 111-158, 1984.

SILVA, S. M. A floresta Atlântica no Paraná. In: FERNANDES, C. R. (Ed.). Floresta Atlântica: reserva da biosfera. Curitiba: UFPR, 2003. p. 17-67.

SOCIEDADE DE PESQUISA EM VIDA SELVAGEM E EDUCAÇÃO AMBIENTAL. Projeto de restauração da floresta Atlântica. Curitiba, 2000. 17 p.

TOLEDO, L. O.; PEREIRA, M. G.; MENEZES, C. E. G. Produção de serapilheira e transferência de nutrientes em florestas secundárias localizadas na região de Pinheiral, RJ. Ciência Florestal, Santa Maria, v. 12, n. 2, p. 9-16, 2002.

VALENTI, M. W.; CIANCIARUSO, M. V.; BATALHA, M. A. Seasonality of litterfall and leaf decomposition in a cerrado site. Brazilian Journal of Biology, São Carlos, v. 68, n. 3, p. 459-465, 2008.

VARJABEDIAN, R.; PAGANO, S. N. Produção e decomposição de folhedo em um trecho de Mata Atlântica de Encosta no município de Guarujá, SP. Acta Botanica Brasilica, Feira de Santana, v. 1, n. 2, p. 243-256, 1988.

VOGEL, H. L. M.; SCHUMACHER, M. V.; TRÜBY, P. Avaliação da devolução de serapilheira em uma Floresta Estacional Decidual, em Itaara, RS, Brasil. Ciência Florestal, Santa Maria, v. 17, n. 3, p. 187-196, 2007.

WALKER, B. H. Biodiversity and ecological redundancy. Conservation Biology, Santa Barbara, v. 6, p. 18-33, 1992.

Cerne, Lavras, v. 18, n. 1, p. 75-86, jan./mar. 2012 
WERNECK, M. S.; PEDRALLI, G.; GIESEKE, L. F.

Produção de serapilheira em três trechos de uma floresta semidecídua com diferentes graus de perturbação na
Estação Ecológica do Tripuí, Ouro Preto, MG. Revista Brasileira de Botânica, São Paulo, v. 24, n. 2, p. 195-198, 2001.

Cerne, Lavras, v. 18, n. 1, p. 75-86, jan./mar. 2012 